\title{
Fast controls for the WITCH Penning trap radioactive source experiment
}

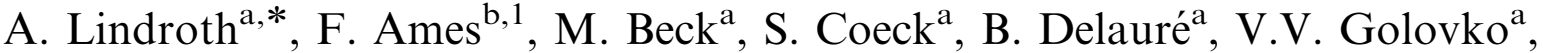 \\ V.Yu. Kozlov ${ }^{a}$, I.S. Kraev ${ }^{\text {a }}$ T. Phalet ${ }^{\mathrm{a}}$, W. Quint ${ }^{\mathrm{c}}$, N. Severijns ${ }^{\mathrm{a}}$, B. Vereecke ${ }^{\mathrm{a}}$, \\ S. Versyck ${ }^{\mathrm{a}}$, the ISOLDE ${ }^{\mathrm{d}}$, EUROTRAPS and NIPNET collaborations \\ ${ }^{a}$ Instituut voor Kern- en Stralingsfysica (IKS), Katholieke Universiteit Leuven, Celestijnenlaan 200D, B-3001 Leuven, Belgium \\ ${ }^{\mathrm{b}}$ Ludwig-Maximilians-Universität, Am Coulombwall 1, D-85478 Garching, Germany \\ ${ }^{\mathrm{c}}$ GSI-Darmstadt, Planckstr. 1, D-64291 Darmstadt, Germany \\ ${ }^{\mathrm{d}} \mathrm{CERN}, \mathrm{CH}-1211$ Genève 23, Switzerland
}

Received 17 May 2004; accepted 15 June 2004

Available online 20 August 2004

\begin{abstract}
The WITCH experiment will for the first time use a dedicated Penning trap as a radioactive source. By combining this with a retardation spectrometer, general access is opened to the $\beta$-recoil energy observable, which allows, e.g. to test the validity of the V-A form of the weak interaction. A system of specialized electronics with accompanying networked software control system has been developed for WITCH. Herein is given a short overview of the general control system and details about the electronic as well as software components special for the fast control of this Penning-trap-plusspectrometer set-up.
\end{abstract}

(C) 2004 Elsevier B.V. All rights reserved.

PACS: 07.05.Dz; 23.40.Bw; 29.30; 29.25

Keywords: Control system; Front-end electronics; Interlock; Penning trap

\footnotetext{
*Corresponding author.

E-mail address: axel.lindroth@fys.kuleuven.ac.be (A. Lindroth).

${ }^{1}$ Present address: TRIUMF, 4004 Wesbrook Mall, B.C., Canada V6T 2A3.
}

\section{Introduction}

The measurement of $\beta$-recoil spectra has long been severely hampered by energy loss and complete stopping of the recoiling ions already in the source. Ref. [1] reports the first attempt to use as radioactive source an ion-cloud suspended in a Penning trap, to avoid the similar but much less-pronounced scattering problem for conversion 
electron spectroscopy. As described in more detail in previous publications [2,3] the WITCH set-up (Weak Interaction Trap for Charged particles) at the ISOLDE facility in CERN uses a dedicated Penning trap to allow to fully exploit and optimize it for use as a radioactive source, mainly for $\beta$-recoil ions. Also discussed in those references is the physical information that can be extracted from the recoil spectrum. It suffices here to mention only the paramount example provided by the Lorentz structure of the weak interaction. The shape of the recoil spectrum is predicted by the Standard Model vector and axial vector contributions to the weak interaction causing the $\beta$-decay. Any exotic contributions, such as scalar or tensor currents, to the Standard Model "V-A" form will modify the shape of the recoil spectrum. It is the main aim of WITCH to improve our knowledge about such currents.

The experiment (see Fig. 1) is roughly $10 \mathrm{~m}$ long, starting at the REXTRAP [4] beam preparation Penning trap and ending, after bending $90^{\circ}$, just underneath the ceiling of the ISOLDE hall. Between those ends there are four sections; beam transport, retardation and injection, a trap section with a double Penning trap structure, and a retardation spectrometer. Radioactive ion bunches are delivered by REXTRAP, and transported at $60 \mathrm{keV}$ energy to the vertical beam-line section where they are retarded in steps. A long pulsed drift tube (PDT), over which the voltage is ramped by $\sim 60 \mathrm{kV}$ while the ions pass through it, is used for one of those steps. This system allows reducing the ion beam energy before injection into the traps, without the need to elevate a section of the set-up on high voltage. The lower trap is used to catch and mass selectively cool the radioactive ions, after which they are transferred to the upper trap where their decay is studied. The energy spectrum of the recoiling ions is collected by scanning a retardation voltage and counting on a micro-channel plate detector (MCP) the number of ions that pass the potential barrier. Two super-conducting solenoids, housed in a common cryostat and manufactured by Oxford Instruments, create a strong homogenous field (9T) for the Penning traps as well as a field gradient for converting radial energy into axial. Since the retardation voltage probes only the latter, as complete conversion as possible is

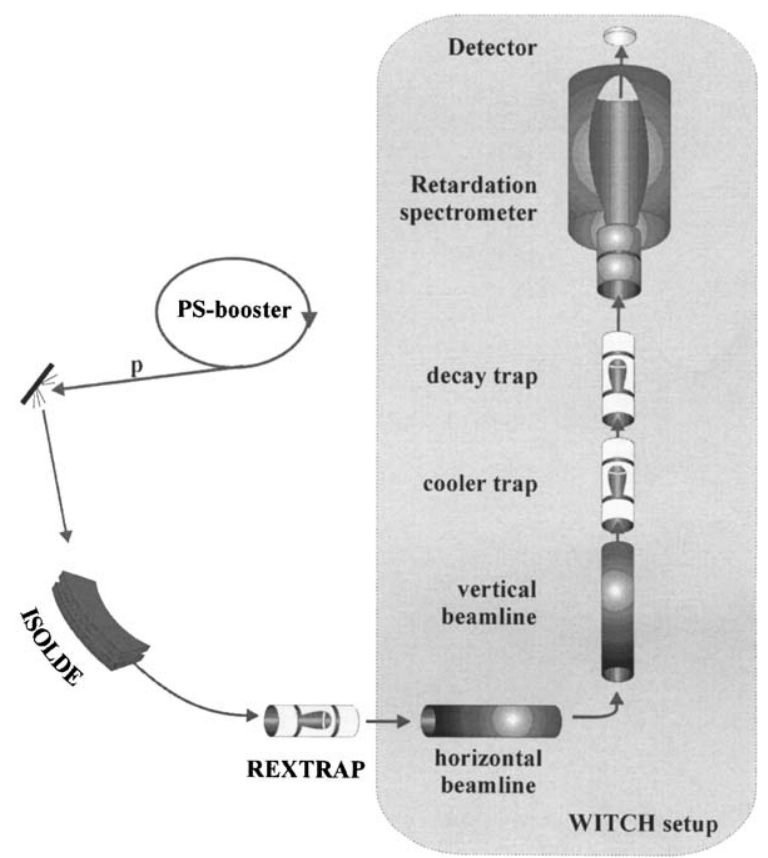

Fig. 1. Layout of the WITCH experiment at ISOLDE, CERN. All sections except the transport section (horizontal beam-line) are actively controlled in the experimental cycle. The retardation steps discussed in the text are located in the vertical beam-line.

desirable, requiring that the magnetic field changes adiabatically along the ion motion.

A dedicated system of electronics and software controls has been developed, partly within the European RTD network NIPNET. The complete control system consists of a standard slow control part, an experiment-specific fast control part for the trap-and-decay cycle, and software with both the slow and fast controls as well as data acquisition integrated. In this report we give a minimal introduction to the general part of the control system, but focus more attention to the experiment-specific fast control. A typical cycle of experiments with radioactive ions in Penning traps is described, and the requirements this puts on the control system are mentioned.

\section{General description and slow control}

The complete control system consists of electronic device controllers, a few different bus systems 
that connect these controllers to a network of computers, as well as the networked software system running on those computers. This software allows in principle full control of all device controllers in the experiment. It integrates the slow and the fast, specialized controls, as well as the data acquisition, and is described in Section 4. In terms of the number of parameters to control, which is of the order of 1000 (see Table 1), this system is small. However, special operating requirements during the combined trapping and spectrometer measuring cycle (fast cycle) have necessitated new developments in both hardware and software controls. The front-end of the computerized control system consists, in a first version, of only two personal computers (PCs), of passive-backplane type. Such PCs have many PCI and ISA buses available, and thus allow many devices and controller cards to be installed on the same computer (in our case: 18 serial ports, GPIB, ISOBUS, CAN, digital $\mathrm{I} / \mathrm{O}$, analogue $\mathrm{I} / \mathrm{O}$, scalers, a multi-channel analyzer, and a pattern generator.) This is advantageous for an experiment for which the software is developed at another location than where the experimental set-up is located since, albeit only one or a few machines have to be transported between the two sites, all buses and other $\mathrm{I} / \mathrm{O}$ channels are in fact carried with them. Access to these channels is absolutely necessary

Table 1

Parameter types and estimates of the number of parameters in the system

\begin{tabular}{lll}
\hline Parameter type & $\begin{array}{l}\text { Number of } \\
\text { parameters }\end{array}$ & Bus type(s) \\
\hline Vacuum & 100 & Serial \\
Cryostat & 30 & GPIB \\
Voltages & 100 & $\begin{array}{l}\text { CAN, ISOBUS, } \\
\text { analogue out }\end{array}$ \\
Beam diagnostics & 100 & $\begin{array}{l}\text { DIO, GPIB } \\
\text { Serial }\end{array}$ \\
$\begin{array}{l}\text { Fast Cycle } \\
\text { Other }\end{array}$ & 200 & $\begin{array}{l}\text { Serial } \\
\text { Controller Aux }\end{array}$ \\
& 500 & $\begin{array}{l}\text { All the above- } \\
\text { mentioned types }\end{array}$ \\
\hline
\end{tabular}

The fast cycle parameters consist of trap DC voltages $(\sim 100$ parameters), trap RF parameters $(\sim 20)$, timing parameters $(\sim 40)$, and retardation power supply ( $\sim 85$ parameters). The last row represents signals that change the state of devices, check their status and perform other similar tasks. during the software development in order to test the software functionality, especially for development of instrument driver routines. The software constructed implies no limitations on the number of machines that constitute the front-end of the control system. All PC's run Windows $2000{ }^{\circledR}$.

The bulk of standard, slow controls are in the vacuum control units, beam-line electrodes' power supplies, beam diagnostics, and super-conducting magnet control. Full control of the vacuum is implemented via the electronic controllers of turbo pumps, vacuum gauges, and valves. The two former use serial bus communication, and the valves are controlled by voltages generated by a controller developed at IKS, which communicates via an ISOBUS. Static electrode voltages are controlled via three bus systems: a CAN fieldbus, ISOBUS, and via analogue signals. Preference has been put on performance of the power supplies themselves, the buses they support have not been a selection criterium. Twelve linear and three rotary vacuum feedthroughs are moved by stepper motors (Tamagawa TS31) with a 15-channel drive made at IKS. They are mostly positioned in pairs, with a collimator strip attached to the upstream feed-through and a Faraday cup (FC) and an MCP on the downstream feed-through. The FC is read out by an electrometer (Keithley 6512) and ancillary switching unit (Keithley 7001 switching mainframe plus 10-channel switching card Keithley 7158). Since some FCs are located in drift sections that are not on ground potential, the readout is capacitive in order to allow the signal to be read out from ground although the FCs are on high voltage. For the super-conducting double magnet cryostat, controllers have been developed at IKS for the multitude of diagnostic sensors available. Most of these sensors are temperaturedependent resistances. Oxford instruments power supplies and level meters are connected to the GPIB bus, whereas the IKS controllers are on individual serial buses.

\section{The experimental cycle and fast control}

In WITCH, fast control is needed for the following pieces of instrumentation: (1) the PDT, 
(2) the two Penning traps, (3) the spectrometer retardation steps, and (4) the detection electronics for the counting MCP. For the first two pieces of instrumentation the information herein resembles that of other set-ups using radioactivity in Penning traps, and the discussion applies rather generally. An experimental cycle consists very roughly of injection into the lower trap, manipulation of the ion cloud, transfer into the second trap, a spectroscopic study of the decay, and ejection of the remaining ions. The cycle time is in principle determined by the half-life of the isotope studied. For the nuclides suitable for improving limits on scalar contributions to $\beta$-decay the half-lives are one to a few seconds $[2,3]$. The cycle time is also affected by limitations inherent in the experimental method, and half-lives much below one second most likely pose too big difficulties, due to, e.g. transport and cooling times. In the present technical solution there is, however, a more stringent limitation of the cycle time $\gtrsim 1 \mathrm{~s}$ due to details in the retardation section as mentioned below. There is also an upper limit on the duration of a cycle due to the limited time that a large ion cloud can be stored in a Penning trap.

The fast control cycle is timed by a pattern of the type shown in Fig. 2. The experimental cycle of WITCH is started by the ejection of an ion bunch from REXTRAP. Such bunches can be delivered at up to $50 \mathrm{~Hz}$, but since the cycle time of WITCH corresponds to $\leqslant 1 \mathrm{~Hz}$ REXTRAP will be run at a corresponding frequency. A trigger signal from the REXTRAP set-up starts a one-shot run of a timing pattern in the fast control of WITCH. Note that some of the steps in Fig. 2 consist of substeps, which increases the number of timing channels needed. E.g. during injection of the ions into the first Penning trap a number of voltages may be raised or lowered at different times. In the cooling phase radio-frequency fields are applied to excite and mass-selectively cool and center the ions. During measurement the retardation voltage sweep is stepped through and the detection electronics is gated for each step. Finally, the remaining ions in the decay trap are ejected and the system is set in ready-state to await a new pulse

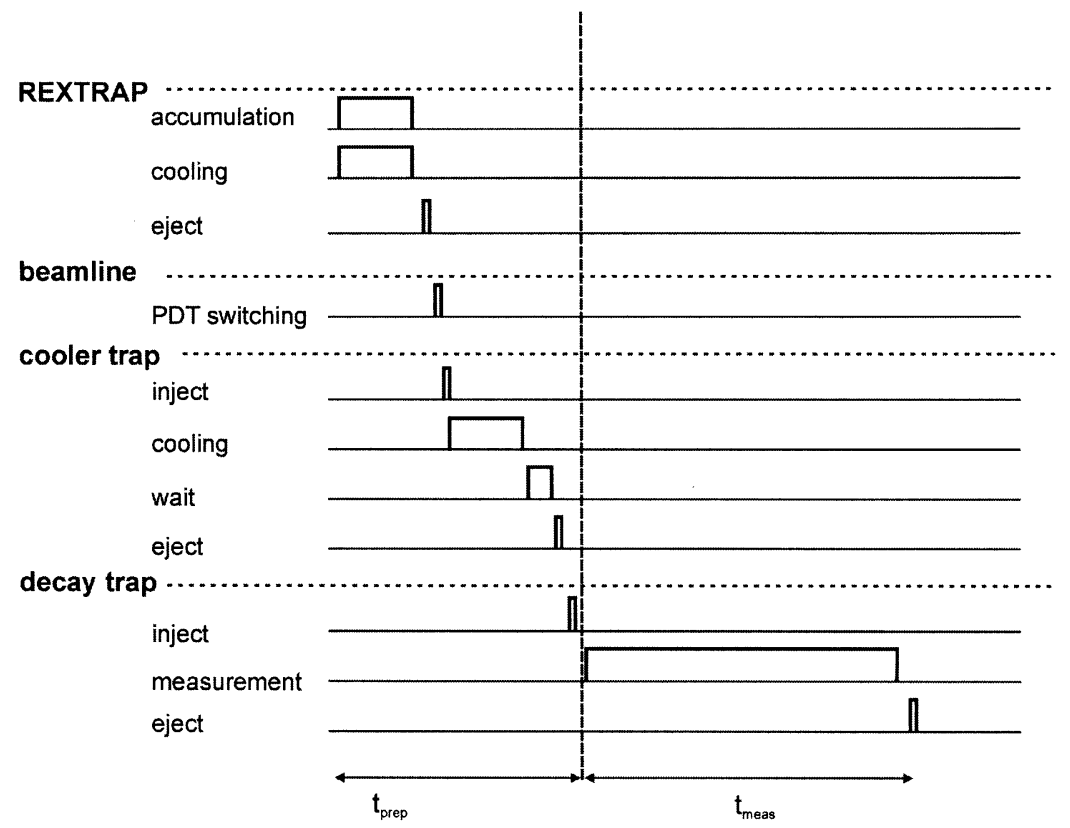

Fig. 2. Timing pattern of a typical experimental cycle. Each line except the "measurement" trigger is in its logical 1 state during some tens of ms. The measurement part of a cycle will typically be one to a few seconds long. This pattern is created by a combination of the PPG100 pattern generator board and a 20-channel gate- and delay generator (GaDG) specifically tailored to the WITCH requirements. 
to be provided by REXTRAP. After the initiation of the WITCH cycle by the REXTRAP TTLtrigger, the timing pattern that follows controls the four pieces of instrumentation, mentioned above, via electronic devices. For the most part these had to be developed for this set-up, although some of the necessary devices have been possible to acquire commercially. Fig. 3 shows the electronic devices that implement the fast control, and how they are connected to the instrumentation and computer network. Note that, due to the fact that the triggering of the detection electronics has to be synchronized with the Penning trap cycle, which re-fills the radioactive source, it is necessary that the data acquisition and control system are integrated.

Penning traps allow the use of certain ion manipulation schemes, which include the application of external dipole or quadrupole fields of either one of the three eigenfrequencies of the ion motion: axial, magnetron, and reduced cyclotron motion, as well as of the true cyclotron frequency. For example, the cooling phase indicated in Fig. 2 entails the application of an azimuthal quadrupole field at the true cyclotron frequency $[5,6]$. In many applications this cooling is preceded by a dipole excitation at the magnetron frequency, which will increase the radius of this motion independently of ion mass. The above-mentioned cooling, which also centers the ions, is mass selective and the combined excitations therefore make sure that only ions of a certain mass are present in the center of the trap. The timing is important since, e.g. the radius of the magnetron motion increases linearly with the time that the dipole field is applied, and naturally the timing of voltage changes in the traps relative to the timing of events in other sections of the set-up are crucial too. The time extension of the ion bunch that passes the PDT and is injected into the lower trap is a few $\mu$ s, and also the time it takes for this bunch to be moved between different parts of the set-up, e.g., from the PDT to the lower trap, is of the $\mu$ s order. Because of this the timing modules must have a resolution clearly below $1 \mu \mathrm{s}$. Furthermore, the jitter should be low enough to avoid that any systematic problems arise, especially in the timing of the retardation voltage supply described in the end of this section.

For timing of events in the experimental cycle a combination of a commercially available

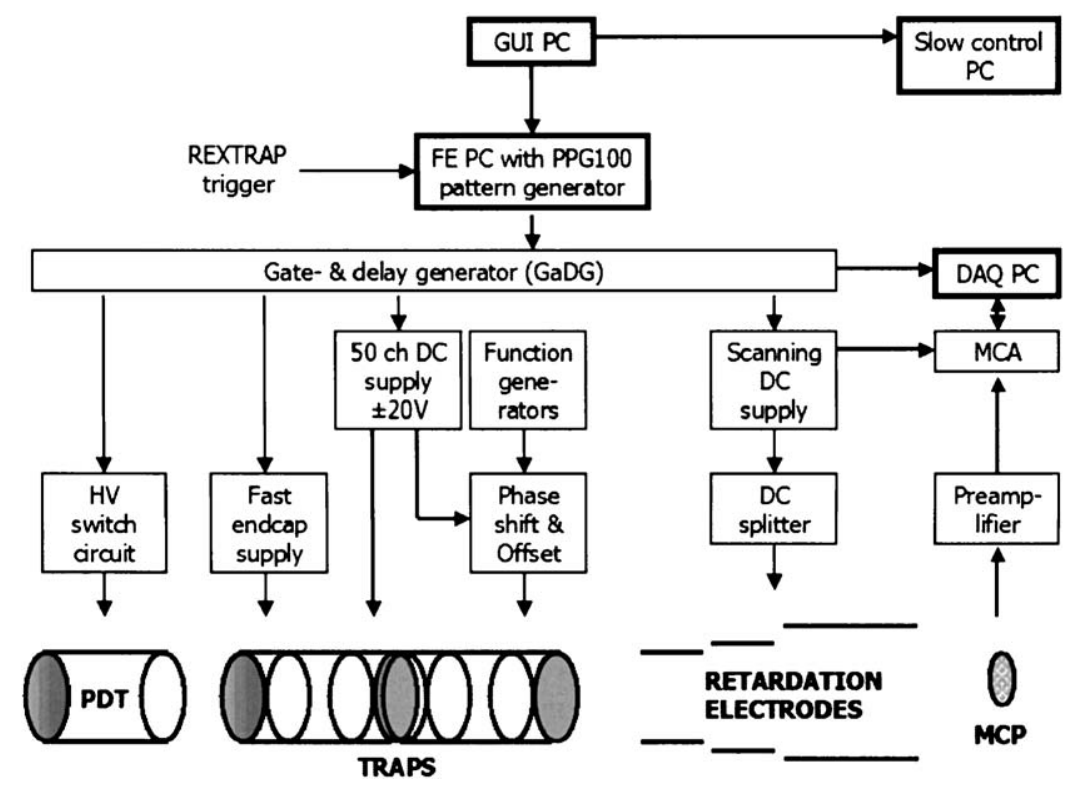

Fig. 3. The connections of the electronic devices implementing the fast cycle. Timing signals are shown, but the direct control of parameters in function generators, scanning DC supply, etc., that the front-end (FE) PC executes, are omitted. 
32-channel pattern generator (Becker and Hick1 PPG100, connected to the passive-backplane ISA bus) and a multi-channel gate- and delay generator (GaDG) specifically tailored for the WITCH requirements, and developed at IKS, are used. The pattern generator is more versatile than a simple GaDG and allows individual signal lines to be switched off and on (logical 0 or 1) several times during each cycle, which is useful e.g. for triggering the stepping of the retardation power supply described below. It also offers logical starting and re-starting (looping) conditions on its four TTL input lines. This feature is used to trigger the WITCH cycle on the REXTRAP ejection signal, which is the base time of every cycle, and all other event timings are relative to this. The pattern file of the PPG100 pattern generator is uploaded once and for all when suitable settings have been found for an experiment. For time scans during optimization or experiment the GaDG is therefore used.

The GaDG has been designed to allow scanning of many delay values over ranges not commercially available. It has a $100 \mathrm{~ns}$ precision from 0 to $429 \mathrm{~s}$ (the maximum is determined by the use of a 32 bit counter), with $15 \mathrm{~ns}$ jitter. Each of the twenty channels has an independent gate and input for an external clock as well as both a gate and a delayed gate output. The gate/delay time of each channel is set via the serial bus from a front-end PC.

In the following we describe the hardware controls and specialized electronics that are triggered by the above-mentioned timing modules, starting from the bottom of the set-up. The voltage switching of the PDT in the retardation section (vertical beam-line; Fig. 1) is not straight-forward as only a limited peak current can be accepted over the circuit containing the high-voltage switch (Behlke hts650-03-lc), from which a few $\mu$ s switching of up to $65 \mathrm{kV}$ is required in order to not loose part of the radioactive ion-bunch. In addition to the pulsing down of the voltage with a fall-time in the $\mu$ s regime, it is also important that the switch can regain its initial voltage with a rise-time that doesn't restrict the fast cycle time too much. With the present switch the limit caused by the rise-time is around $1 \mathrm{~s}$. Similar switching issues have been addressed also in other Penning trap set-ups working with radioactive ions, Ref. [7]. There, further developments have been needed to reduce a leakage current that was measured through the switch after some time of use. The implemented solution uses two switches in series [8]. A serial connection of two $30 \mathrm{kV}$ switches as was used is not optimal in our case since this will not reduce the time constant of the exponential decay of the switching. A future improvement of the PDT pulsing in WITCH is being considered, and entails the use of two $65 \mathrm{kV}$ switches in parallel, by which the switching time can be reduced by a factor of two.

The voltages on the trap electrodes are produced by a pair of function generators (Stanford Research DS345), a DC power supply built at IKS, and an 18-channel box that offsets the RF signals from the DS345 by an input DC voltage and gives a $180^{\circ}$ shifted signal out in addition to the non-shifted one. The shifted signals are needed for applying the RF quadrupole and dipole fields on the ring electrodes of the two traps, as well as for certain ion-cloud manipulation techniques, e.g., the "rotating wall" technique [9], which can be used to overcome the space-charge repulsion in order to increase the ion density in the cloud. Such increase in density is directly related to the maximal intensity that the radioactive source can have. The offset voltage of the RF signals is delivered by a two-level DC supply that can deliver between $\pm 20 \mathrm{~V}$ on 50 channels. Fast switching between the two levels allows to use different voltages for capture and storage of ions. This supply is also used for most of the trap electrodes where no RF field is applied. The outer, so-called "end-cap" electrodes of each Penning trap need to be pulsed up and down fast (one to a few $\mu$ s) for capture and ejection of the radioactive ions in the Penning trap [10]. This pulsing should be between 0 and a much higher voltage than the $20 \mathrm{~V}$ that suffices for the other trap electrodes, since the kinetic energy of the ions will be several tens of $\mathrm{eV}$ and will have a large uncertainty even when very precise power supplies are used in the retardation steps. The lower end-cap is on the same potential as the trap center as the ion bunch arrives, but as the ions pass into the trap the voltage must be switched fast in order to contain 
them. A seven-channel power supply which allows the fast switching of up to $+150 \mathrm{~V}$ has been specifically tailored for Penning trap applications by the GSI contingent of the NIPNET European RTD network [8].

Concerning the spectrometer section, the retarding voltage can be changed either only between cycles, or swept over its whole range within each cycle. This would correspond to a constant retardation voltage during each trap filling, or a fast stepping of the voltage during each filling, respectively. The latter is naturally preferable to avoid systematic errors. A power supply has been developed for the special requirements of the retardation electrodes. The voltages are set by this one supply, and are divided by a potentiometer chain so that the slow increase in voltage over the series of electrodes results in adiabatic motion (see Section 1). The supply itself is designed to step through a number (1-40) of voltages within each measurement cycle. After the time it takes for the voltage to stabilize at a value sufficiently close to the set value of each step, a TTL trigger pulse is put on an output line from the supply. This trigger is switched to logical zero before the next step is initiated. Detection electronics is gated by this signal to make sure that data is only collected on well-known voltage values. By such a scheme of voltage stepping, it is ensured that the entire recoil spectrum can be measured within each experimental cycle, thus avoiding difficult systematic issues and normalization of the number of ions in the ion cloud at each trap filling. The supply is specified to staying $10 \mathrm{~ms}-1 \mathrm{~s}$ on each step, and the time between steps, "dead time", should be minimized (it is $\sim 10 \mu \mathrm{s}$ ). The precision of the voltage of each step is limited to $\pm 0.1 \%$, but this is anyway small compared to other sources of widening of the response function. An additional advantage of this triggering scheme is that the detector electronics is only counting ions during the period when the decay-trap has a reasonable amount of un-decayed nuclei. Like this, full advantage is taken of the background reduction that is related to the use of a bunched beam. This second advantage is present regardless if the retardation voltage is swept during each cycle or only changed between cycles.
The MCPs, which are used for beam diagnostics as well as for detection of the recoil ions in the spectrometer, deserve a special comment here. Up to, but not including the PDT the set-up can in principle accept ion bunches from REXTRAP at $50 \mathrm{~Hz}$. Since the high voltage switching of the PDT needs towards $1 \mathrm{~s}$ to regain the initial voltage after being pulsed, the ion bunches can not arrive at a higher frequency than $\sim 1 \mathrm{~Hz}$ from there on. This makes readout less straightforward since a Faraday cup read-out in an integrated mode will not allow high enough precision with the Keithley 6512 electrometer used. Count rates of the order of $10^{11} \mathrm{cps}$ occur during $10 \mu \mathrm{s}$ as a short ion bunch with $10^{6}$ ions hits the beam diagnostics detector. For this case the readout electronics for eventcounting in an MCP is completely saturated giving a total detection efficiency of around $10^{-4}-10^{-5}$ for an electronic deadtime of $15 \mathrm{~ns}$ [11]. At these higher count rates an integrating mode must be implemented, e.g by using an oscilloscope or electrometer. Alternatively, the number of ions detected in the MCP can be limited by e.g. defocusing. The latter solution is less useful since it implies modified beam properties. Integration of the oscilloscope signal is the method in use at WITCH. In the recoil detector the situation is similar because of the high intensity in the early stage of an exponential decay. The possibility of de-focussing is naturally not an option there. However, by using a large-area detector with segmented anode, and not focusing but rather to use an Einzel-lens, which is located just before the detector, to spread the recoil ion events over the entire MCP surface, a reduced counting rate is achieved which allows longer time for the MCP channels to recover.

\section{Software architecture}

For the experimentalists' convenience a set of computer programs has been developed to control the experimental parameters. The software has much in common with the "CS" control system environment described in Ref. [12], and much technical information has been exchanged via the NIPNET network mentioned above. Specifically, 
the software requirements are very similar, see p. 6 of Ref. [12]. In short, the six requirements listed in that work are (1) data acquisition and Supervisory Control And Data Acquisition (SCADA) capability should be included, (2) a Ph.D. student must be able to maintain and further develop the system after a few months of learning time, (3) the system should be distributed and allow remote access, (4) full and immediate control of all electronic devices in the system, (5) inclusion of new versions or additional device drivers must be easy, and (6) the system must be stable since beam-time is limited. The CS software is general and can be easily applied to different experiments, and here lies the biggest difference to the WITCH software. The latter has no generality and is adapted only to WITCH. By that it has been possible to minimize development time and put extra emphasis on the second requirement above. The amount of program code has been kept as small as possible for the same reason, and only one software package $\left(\mathrm{LabView}^{\circledR}\right)$ is used. Since LabView has a dataflow architecture, the software has been developed in a modular fashion rather than object oriented. The LabView programming environment has been used mainly because of the time saved in driver development, since many $\mathrm{I} / \mathrm{O}$ cards are available with drivers for LabView. SCADA capacity is implemented using the "Datalogging and Supervisory Control" engine (DSC $\left.{ }^{\circledR}\right)$ module of LabView. This module runs as a separate process and can store data in channels ("Tags") on hard disc or temporarily in the random access memory ("Memory Tags"). The DSC software includes alarms that are used to warn the user when there are problems with parts of the set-up that potentially could cause danger to people, equipment, or to the integrity of data.

Both slow and fast parameters are controlled from the same software system. There are two types of slow control signals: (1) those that concern the control of pumps and of similar devices, where in principle no changes are needed during an experiment, and (2) those that set the parameters of the fast, hardware-run control. The software developed integrates these parts, but here we discuss only the software for the second type of slow control and for the fast control, as well as general features of the software.

The programs operate at three network layers: a local network, the CERN network, and the internet. At both borders a gateway with firewall keeps the layers apart to the necessary degree. The whole networked system is partly event-driven, partly carries out periodic tasks, and the eventdriven side can be used to change the settings of the periodic part. Graphical user interfaces (GUIs) allow the user to access any parameter of any device over a messaging system-this is initiated by the user. (GUIs can be run on any machine in the network, and in special versions implementing a higher level of security also over the internet.)

The processes of the system are indicated in (Fig. 4), and are described in the following. In addition to the DSC engine there is a TCP/IP daemon that routes messages between processes, a sequencer process, an interlock process, and a number of instrument control processes. On the machines running GUIs there is also a "Main GUI". This process consists of ancillary functions for starting up all processes of the system, and a watchdog to provide warnings if a process should stall. It also displays warning messages appearing in a tag dedicated to errors in the software system.

Inter-process communication is provided by a daemon combining the handling of message-based TCP/IP sessions on the network and messagequeues on the local machine (in Fig. 4 this process is refered to as "Communication"). The messages pass directly via message-queues only for messages between processes on the same machine, and for messages between different machines the communication process routes the incoming TCP/IP message to the appropriate queue on the receiving machine. Any computer to be used in the system needs a running instance of this process. TCP/IP messaging is used to achieve maximal flexibility as to which communications can be implemented. In some cases a simplified implementation using the National Instruments DataSocket server has been used, especially for data that is continuously published to several GUI machines. The DataSocket server, which bridges the transport and application network layers, accepts multiple 


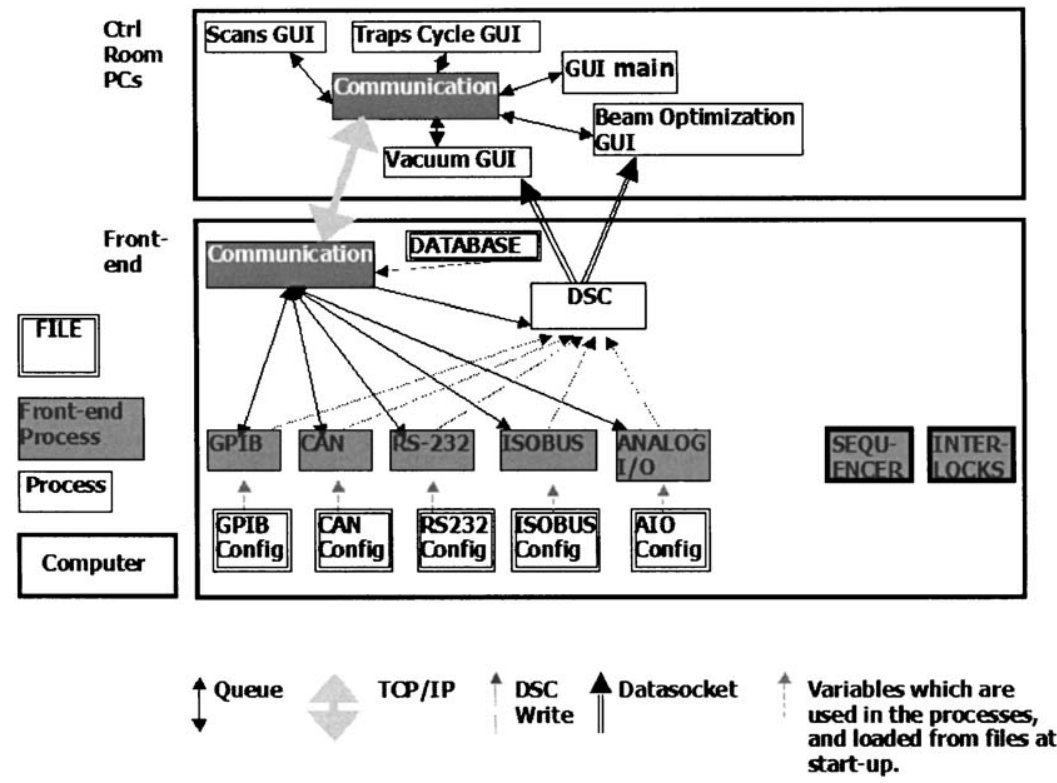

Fig. 4. Generic software architecture of the integrated fast and slow control system. The number of machines and processes has been reduced to make room for more detail, and for clarity the communications of the sequencer and interlock processes have been left out. The Communication processes handle the local message-queue administration and the TCP/IP connections to other machines. Data is transferred via DataSocket connections when a continuous overview of many parameters is published to several machines without direct action by the user.

connections unlike the basic TCP/IP upon which this higher-level session-handling software is based. Each process is implemented as a queued message handler, with an input queue and an output queue handled by separate threads. A database is kept with information about which driver or core process is running on which machine. (Not so for GUIs - no other process will contact a GUI without first receiving a message from that GUI, and any number of copies of each GUI can be running on different machines in the network. GUIs are the sources of events in the event-driven part of the system.) Using this database, messages can be addressed directly to a process, which allows processes to be easily moved between machines for optimization of the system. This is particularly the case for instrument control processes, described below.

The instrument control processes are multithreading, and one of the threads handles periodic events, like checking important parameters of the device it controls and writing them to the DSC process for logging to the hard disc. In this system we naturally include mainly those parameters that could be needed in the data analysis to check e.g. stability of electronics. A second thread converts messages on the in-queues of the process into commands for the appropriate device controller on the corresponding bus. In modularizing the instrument control processes the deciding criterium has been whether a device can be communicated with independently of any other ongoing communication. For example, the vacuum gauge control units allow read-out of pressures via the serial interface, but only of one of its gauges at a time, and therefore each controller has its own program rather than one for each gauge. Each bus in Fig. 4 represents a number of independent instrument control processes communicating on that bus.

The interlock process analyzes the relevant parameter values that are read back periodically from the electronic controllers, and implements a more complex warning system than the DSC 
engine, which only checks that a parameter is within its allowed range. It can e.g. make the allowed range depend on the value of another parameter in the system, which is relevant in the case of two neighboring vacua with a separating valve. The interlock process can write a warning message in a dedicated tag logged by the DSC engine, which is periodically read by the "main GUI" on each control-room machine. It can also take the necessary action independently, e.g. to close a valve or ramp down a voltage, and in addition it prevents the user from setting illegal values of parameters.

The sequencer process handles the changing of parameter values between experimental cycles, and it sets the PPG100 board in a state where it can be triggered by the REXTRAP ejection signal only when the new parameter values have been verified. The main use is to make scans. The scans are in general requested from the GUI layer via the messaging system, and loops through a number of delay values (or other parameters), reads back that they have been correctly set, and reads the value of the scanned parameter (e.g. the number of detected ions). Scans are needed for optimization of both fast and slow parameters before experiment, but also if for some reason one would prefer to run an experiment without sweeping the retardation voltage within each cycle. Then, the sequencer process checks that the retardation voltage has stabilized on the value set for the next counting period, before starting that measurement cycle. For the retardation stepping DC supply such sequencing is not necessary, and therefore it is expected to be mainly used for scans collected as a function of other parameters than the retardation voltage. Another possible use of this process is to check that the PDT switch circuit's capacitors have been re-charged so that the full retardation voltage is present on the PDT. Optimization of the cycle times is done by scanning versus different delay times in the GaDG.

A database provides an abstraction layer within the messaging system. From the GUIs, messages contain information about in which part of the setup, e.g. a voltage, should be changed. This information is converted into a specific device and channel in the abstraction layer.

\section{Conclusion}

A capable control system is at hand for the WITCH experiment at the same time as the construction of the experiment itself is being finished. Its implementation allows an efficient use of WITCH in fundamental physics measurements already from the start-up. Flexibility is assured as maintenance and upgrades are as simple as possible due to the limited size of the software code. The slow control will be equipped with more extensive readback possibilities and some more intelligent processes in a next development phase. Read-back of beam-line electrode voltages and automated beam tuning are developments of this type that would be helpful. For the fast control most developments will aim at improving the user-friendliness of the software controls. Real-time reading back of the voltages on the surface of the retardation electrodes is being considered. This would also include a comparator to check that the read back value is the same as the set value before the MCP is triggered.

\section{Acknowledgements}

This work is supported by the European Union Grants FMRX-CT97-0144 (the EUROTRAPS TMR network) and HPRI-CT-2001-50034 (the NIPNET RTD network), by the Flemish Fund for Scientific Research FWO and by the Project GOA 99-02 of the K.U.Leuven. Additional funding, in terms of a specialization fellowship of the Flemish Institute for the stimulation of Scientific-Technological Research in the Industry (IWT), has been received. Important advice and suggestions from Dietrich Beck, at the DVEE department, GSI, Germany, is gratefully acknowledged. Members of the NIPNET working group for trap control systems are thanked for exchange of ideas and methods as well as development of instrumentation needed.

\section{References}

[1] L. Weissman, F. Ames, J. Äystö, O. Forstner, K. Reisinger, S. Rinta-Antila, Nucl. Instr. and Meth. A 492 (3) (2002) 451. 
[2] A. Lindroth, M. Beck, B. Delauré, V.Y. Kozlov, N. Severijns, F. Ames, D. Beck, V.V. Golovko, I. Kraev, T. Phalet, S. Versyck, Nucl. Phys. A 721 (2003) 1103C.

[3] ISOLDE, EUROTRAPS and NIPNET collaborations, Nucl. Instr. Methods A 503 (2003) 567.

[4] F. Ames, "REXTRAP, an ion buncher for REX-ISOLDE", in: B. Sherrill, D.J. Morrisey, C.N. Davids (Eds.), ENAM98: Exotic Nuclei and Atomic Masses, American institute of Physics, 1998, p. 927.

[5] G. Savard, S. Becker, G. Bollen, H.J. Kluge, R.B. Moore, T. Otto, L. Schweikhard, H. Stolzenberg, U. Wiess, Phys. Lett. A 158 (5) (1991) 247.

[6] M. König, G. Bollen, H.J. Kluge, T. Otto, J. Szerypo, Int. J. Mass Spec. Ion. Proc. 142 (1-2) (1995) 95.
[7] F. Herfurth, J. Dilling, A. Kellerbauer, G. Bollen, S. Henry, H.J. Kluge, E. Lamour, D. Lunney, R.B. Moore, C. Scheidenberger, S. Schwarz, G. Sikler, J. Szerypo, Nucl. Instr. and Meth. Phys. Res. A 469 (2) (2001) 254.

[8] F. Herfurth, private communication.

[9] X.P. Huang, F. Anderegg, E.M. Hollmann, C.F. Driscoll, T.M. Oneil, Phys. Rev. Lett. 78 (5) (1997) 875.

[10] H. Schnatz, G. Bollen, P. Dabkiewicz, P. Egelhof, F. Kern, H. Kalinowsky, L. Schweikhard, H. Stolzenberg, H.J. Kluge, Nucl. Instr. and Meth. Phys. Res. A 251 (1) (1986) 17.

[11] S. Coeck, to be published.

[12] D. Beck, K. Blaum, H. Brand, F. Herfurth, S. Schwarz, Nucl. Instr. and Meth. A 527 (3) (2004) 567. 